CIHR-Thoracic Imaging Network of Canada and the United States Public Health Service contracts N01-CN 35000 from the National Cancer Institute for the OCT technology development. The funding sources for this study played no role in the design and conduct of the study; in the collection, management, analysis, and interpretation of the data; or in the preparation of the manuscript. The funding sources did not review the manuscript. S. Lam had full access to all of the data in the study and takes responsibility for the integrity of the data and the accuracy of the data analysis. Funding information for this article has been deposited with FundRef.

Conflict of interest: Disclosures can be found alongside the online version of this article at erj.ersjournals.com

Acknowledgements: We thank Myles McKinnon, Jennifer Campbell and Chulho Hyun (British Columbia Cancer Agency, Vancouver, BC, Canada) for their assistance with the bronchoscopy procedures.

\title{
References
}

1 Wechsler ME, Laviolette M, Rubin AS, et al. Bronchial thermoplasty: Long-term safety and effectiveness in patients with severe persistent asthma. J Allergy Clin Immunol 2013; 132: 1295-1302.

2 Castro M, Rubin AS, Laviolette M, et al. Effectiveness and safety of bronchial thermoplasty in the treatment of severe asthma: a multicenter, randomized, double-blind, sham-controlled clinical trial. Am J Respir Crit Care Med 2010; 181: 116-124.

3 Hanania NA, Wenzel S, Rosen K, et al. Exploring the effects of omalizumab in allergic asthma: an analysis of biomarkers in the EXTRA study. Am J Respir Crit Care Med 2013; 187: 804-811.

4 Nakano Y, Wong JC, de Jong PA, et al. The prediction of small airway dimensions using computed tomography. Am J Respir Crit Care Med 2005; 171: 142-146.

5 Kauczor HU, Ebert M, Kreitner KF, et al. Imaging of the lungs using 3He MRI: preliminary clinical experience in 18 patients with and without lung disease. J Magn Reson Imaging 1997; 7: 538-543.

6 Coxson HO, Quiney B, Sin DD, et al. Airway wall thickness assessed using computed tomography and optical coherence tomography. Am J Respir Crit Care Med 2008; 177: 1201-1206.

7 Lam S, Standish B, Baldwin C, et al. In vivo optical coherence tomography imaging of preinvasive bronchial lesions. Clin Cancer Res 2008; 14: 2006-2011.

8 Hariri LP, Applegate MB, Mino-Kenudson M, et al. Volumetric optical frequency domain imaging of pulmonary pathology with precise correlation to histopathology. Chest 2013; 143: 64-74.

9 Williamson JP, McLaughlin RA, Noffsinger WJ, et al. Elastic properties of the central airways in obstructive lung diseases measured using anatomical optical coherence tomography. Am J Respir Crit Care Med 2011; 183: 612-619.

10 Coxson HO, Lam S. Quantitative assessment of the airway wall using computed tomography and optical coherence tomography. Proc Am Thorac Soc 2009; 6: 439-443.

11 Tsuboi M, Hayashi A, Ikeda $\mathrm{N}$, et al. Optical coherence tomography in the diagnosis of bronchial lesions. Lung Cancer 2005; 49: 387-394.

12 British Thoracic Society, Scottish Intercollegiate Guidelines Network. British guideline on the management of asthma. Thorax 2014; 69: 1-192.

13 Cox G, Miller JD, McWilliams A, et al. Bronchial thermoplasty for asthma. Am J Respir Crit Care Med 2006; 173: 965-969.

14 Lee AM, Ohtani K, Macaulay $\mathrm{C}$, et al. In vivo lung microvasculature visualized in three dimensions using fiber-optic color Doppler optical coherence tomography. J Biomed Opt 2013; 18: 50501.

15 Whiteman SC, Yang Y, Gey van Pittius D, et al. Optical coherence tomography: real-time imaging of bronchial airways microstructure and detection of inflammatory/neoplastic morphologic changes. Clin Cancer Res 2006; 12: 813-818.

\section{Effective strategies for managing new Pseudomonas cultures in adults with cystic fibrosis}

To the Editor:

Pseudomonas aeruginosa is a common chronic pulmonary infection in cystic fibrosis (CF). It is associated with poor outcomes and new detection should prompt an early eradication attempt [1-3].

Strict segregation and eradication policies have led to a declining prevalence of chronic P. aeruginosa infection, with only $30 \%$ of CF adolescents having chronic infection when they transition to adult services [4]. Adult CF physicians increasingly manage new P. aeruginosa cultures in adults without robust evidence to guide decision-making. We evaluated the success of our eradication and suppression strategies for adults 
with a new or re-emergent $P$. aeruginosa culture in the clinical setting of a large European adult CF centre (Adult Cystic Fibrosis Centre, Royal Brompton Hospital, London, UK).

We undertook a retrospective cohort study of consecutive adult CF patients who were treated with intent to render sputum $P$. aeruginosa-negative after a new $P$. aeruginosa culture in either sputum samples or cough swabs between 2008 and 2012. All patients were diagnosed with CF according to standard published criteria [5]. Patients were included if they had a minimum of three $P$. aeruginosa-negative respiratory samples in the 12 months preceding the treatment period. Patients were categorised into the following groups. Group A: first-ever P. aeruginosa culture; group B: new P. aeruginosa culture and prior history of $P$. aeruginosa-positivity on inhaled antibiotics; and group C: new $P$. aeruginosa culture and prior history of $P$. aeruginosa-positivity but not on chronic inhaled antibiotics. The primary analysis was $P$. aeruginosa culture-negativity success based on sputum taken within 1 month of completing treatment. Subsequent sputum results were recorded from clinic appointments (every 2-12 weeks) up to a minimum of 24 months after the treatment period. At the time of this study, our first-line therapy comprised 3 months of nebulised colomycin $2 \mathrm{MU}$ twice a day and 3 weeks of oral ciprofloxacin $750 \mathrm{mg}$ twice a day; second-line regimens were alternative nebulised (usually tobramycin solution for inhalation (TSI))/oral antibiotic combinations. Second-line regimens were used if the patient was intolerant to, or had previously failed, first-line drugs. For patients already on nebulised colomycin, their first-line regimen was the addition of ciprofloxacin for 3 weeks, and second-line was a switch to an alternative nebulised (TSI)/oral antibiotic combination. In situations where there was evidence of a pulmonary exacerbation, intravenous antibiotics were the preferred option.

New $P$. aeruginosa isolates were identified using our CF departmental database and verified by electronic patient records (EPR). Baseline clinical characteristics and demographics were taken from the UK CF registry, which is collected yearly with informed written consent. Relevant co-existing conditions and medications were recorded from the EPR. Data were analysed using SPSS v22 (IBM, New York, NY, USA). Intergroup variability was tested using an independent samples t-test, ANOVA or a Chi-squared test. We used a $95 \%$ CI and a p-value of $<0.05$ was considered significant.

53 new $P$. aeruginosa cultures (13 in group A, 25 in group B and 15 in group C) from 48 patients (25 (47\%) from female subjects, 45 (85\%) from pancreatic insufficient subjects, 25 (47\%) from patients homozygous for F508del, 13 (25\%) from patients with CF-related diabetes (CFRD)) were included in the analysis. A new $P$. aeruginosa culture was preceded by a median of 6 (range 3-20) P. aeruginosa-negative respiratory samples in the prior 12 months. Baseline characteristics were similar between groups (median $\pm \mathrm{SD}$ forced expiratory volume in $1 \mathrm{~s}(\mathrm{FEV} 1) 65 \pm 21.2 \%$ predicted and body mass index (BMI) $22 \pm 3.2 \mathrm{~kg} \cdot \mathrm{m}^{-2}$ ) although patients in group B were younger (28 (16-54) years in group A, 20 (16-49) years in group B, 29 (17-66) years in group $\mathrm{C} ; \mathrm{p}=0.017$ ). Patients in group $\mathrm{B}$ were most commonly maintained on continuous inhaled colomycin (16 cases) or a monthly alternating inhaled regimen including colomycin and a second agent (TSI) (eight cases).

$28(53 \%)$ patients received our first-line regimen of ciprofloxacin and colomycin, 16 (30\%) patients received i.v. antibiotics, five (9\%) patients received TSI with ciprofloxacin and the remaining four (8\%) received tailored regimens.

Overall, eight $(61.5 \%)$ patients in group A had $P$. aeruginosa successfully eradicated after the treatment period. Eight (61.5\%) patients in the group received first-line treatment, with six (75\%) achieving successful eradication. By 24 months, only two (25\%) patients remained free of P. aeruginosa (fig. 1).

P. aeruginosa culture negativity was achieved in $18(72.0 \%)$ and $11(73.3 \%)$ patients in groups B and C, respectively (nonsignificant). 15 (60.0\%) patients in group B and five $(33.3 \%)$ patients in group C received first-line treatment, with similar proportions achieving culture negativity at 1 month $(73.3 \%$ in group B, $60.0 \%$ in group C; nonsignificant). Of the patients in group B who did not receive first-line treatment, seven $(28.0 \%)$ received i.v. antibiotics and the remaining three (12.0\%) received TSI/ciprofloxacin.

Comparable proportions of patients who received first-line treatment remained culture negative at 24 months (26.7\% in group B, $20.0 \%$ in group C; nonsignificant) (fig. 1). We found no association between the success in achieving $P$. aeruginosa-negative cultures and age, sex, BMI, baseline $\mathrm{FEV} 1$, pancreatic status, co-infection status, CFRD, allergic bronchopulmonary aspergillosis (ABPA) status and mutation class.

Overall patients who had their sputum rendered $P$. aeruginosa negative at 1 month had a median time to re-culturing $P$. aeruginosa of 19.0 months (range 8.3-29.6). There was no significant difference in the time to reacquisition between groups $\mathrm{A}, \mathrm{B}$ and $\mathrm{C}(\log$ rank $\mathrm{p}=0.66)$, or first- and second-line regimens ( $\log$ rank $\mathrm{p}=0.81$ ).

We have shown that sputum can be successfully rendered $P$. aeruginosa-negative in adult patients. Treatment is effective irrespective of first-ever detection versus re-emergence, although the majority of patients re-cultured $P$. aeruginosa within 2 years. 


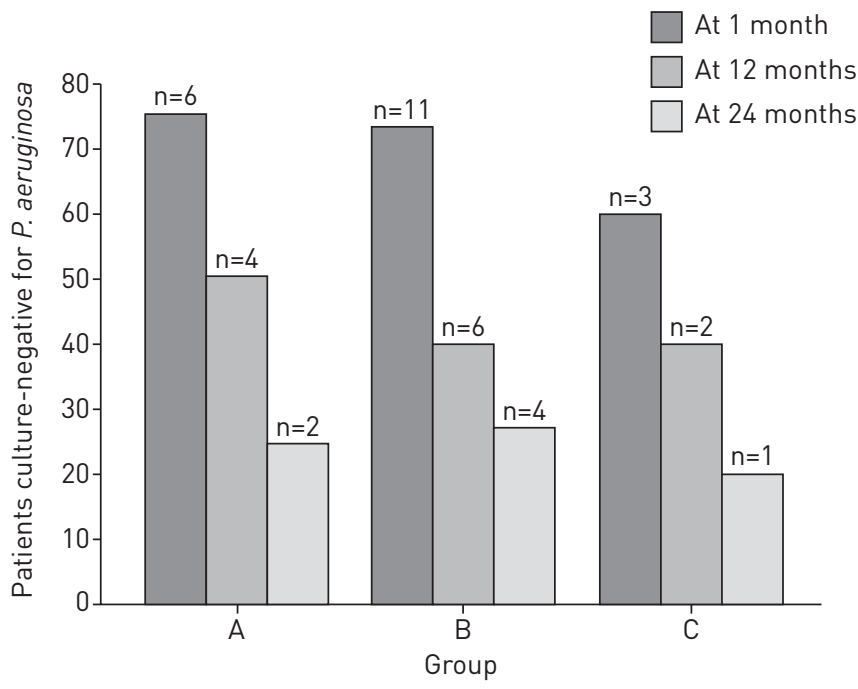

FIGURE 1 Pseudomonas aeruginosa status in patients receiving first-line therapy according to $P$. aeruginosa culture group. Group A: first-ever; group B: re-emergence on inhaled antibiotics; group C: re-emergence off inhaled antibiotics. There was no significant difference in the proportion of patients who were $P$. aeruginosa-free at 1 and 24 months $(\mathrm{p}=0.81)$.

Data reporting the success of $P$. aeruginosa eradication and suppression strategies in adult CF patients are lacking. The only study exclusively of adult patients was retrospective and small $(n=20)$ with a reported eradication success of 79\% [6]. Previous study populations have had milder lung disease, where preserved airway function might facilitate microbial clearance. Our population is older with lower baseline lung function and higher rates of non- $P$. aeruginosa chronic infection ( $75 \%$ had bacterial co-infection), factors which may influence treatment success.

Patients in groups $\mathrm{B}$ and $\mathrm{C}$ represent an increasingly common scenario in adult CF clinics. These are patients who have cultured $P$. aeruginosa in childhood/adolescence but achieve eradication or sustained subclinical suppression into adulthood. It could be argued that group B may represent chronic (but suppressed) infection justifying exclusion from this analysis. Local practice of the paediatric CF unit at our hospital at the time of this study was to have a high threshold for discontinuing inhaled antibiotics in patients with a history of at least two re-emergent $P$. aeruginosa cultures, particularly if they could only provide cough swabs after "eradication" treatment. We believe that including group B, although controversial, adds important information to this debate by demonstrating equivalent rates of sputum negativity regardless of whether this represents true eradication or suppression. A more aggressive approach to a "new" growth in this cohort may be appropriate but longitudinal follow-up is important to determine whether this affects prognostic indices. Measuring P. aeruginosa antibodies may have helped assess whether they had truly eradicated P. aeruginosa in the past [7]. We also acknowledge that measuring time to chronic infection using $P$. aeruginosa antibodies may be a more clinically useful parameter than categorising recurrence as a single $P$. aeruginosa culture. Our patients with $P$. aeruginosa-negative sputum re-acquired $P$. aeruginosa at a median of 19 months, which is similar to paediatric populations [8-10].

Although small, this study provides insight into the effectiveness of our treatments. As CF care advances, more patients will transition to adult services with preserved lung function, free of $P$. aeruginosa cultures. Consequently, eradication and suppression studies in adults are becoming ever more relevant.

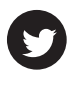

@ERSpublications

Sputum can be rendered $P$. aeruginosa culture-negative in adults with $C F$, although recurrence within 2 years is high http://ow.ly/KPBsY

Haider Ali ${ }^{1}$, Christopher Orchard ${ }^{1}$, Myril Mariveles ${ }^{1}$, Sandra Scott ${ }^{1}$, Khalid Alshafi ${ }^{2}$, Diana Bilton ${ }^{1,3}$ and Nicholas Simmonds ${ }^{1,3}$

${ }^{1}$ Adult Cystic Fibrosis Centre, Royal Brompton Hospital, London, UK. ${ }^{2}$ Dept of Microbiology, Royal Brompton Hospital, London, UK. ${ }^{3}$ National Heart and Lung Institute, Imperial College London, London, UK.

Correspondence: Nicholas Simmonds, Adult Cystic Fibrosis Centre, Royal Brompton Hospital, London, UK.

E-mail: n.simmonds@imperial.ac.uk

Received: June 192014 | Accepted after revision: March 152015 | First published online: April 162015

Support statement: All authors were involved in study design and acquisition of the data. H. Ali and N.J. Simmonds performed the data analysis and drafted the manuscript. All authors were involved in writing the final manuscript. N.J. Simmonds is the guarantor of this manuscript. D. Bilton and N.J. Simmonds are supported by the National Institute for Health Research-funded specialist Respiratory Biomedical Research Unit at the Royal Brompton and Harefield NHS Foundation Trust, and Imperial College London (both London, UK). No specific funding was required for this study. 


\section{References}

1 Nixon GM, Armstrong DS, Carzino R, et al. Clinical outcome after early Pseudomonas aeruginosa infection in cystic fibrosis. J Pediatr 2001; 138: 699-704.

2 Emerson J, Rosenfeld M, McNamara S, et al. Pseudomonas aeruginosa and other predictors of mortality and morbidity in young children with cystic fibrosis. Pediatr Pulmonol 2002; 34: 91-100.

3 Frederiksen B, Koch C, Hoiby N. Antibiotic treatment of initial colonization with Pseudomonas aeruginosa postpones chronic infection and prevents deterioration of pulmonary function in cystic fibrosis. Pediatr Pulmonol 1997; 23: 330-335.

$4 \quad$ UK Cystic Fibrosis Registry. Annual Data Report 2012. Kent, Cystic Fibrosis Trust, 2013.

5 De Boeck K, Wilschanski M, Castellani C, et al. Cystic fibrosis: terminology and diagnostic algorithms. Thorax 2006; 61: 627-635.

6 Kenny SL, Shaw TD, Downey DG, et al. Eradication of Pseudomonas aeruginosa in adults with cystic fibrosis. BMJ Open Respir Res 2014; 1: e000021.

7 Kappler M, Kraxner A, Reinhardt D, et al. Diagnostic and prognostic value of serum antibodies against Pseudomonas aeruginosa in cystic fibrosis. Thorax 2006; 61: 684-688.

8 Taccetti G, Campana S, Festini F, et al. Early eradication therapy against Pseudomonas aeruginosa in cystic fibrosis patients. Eur Respir J 2005; 26: 458-461.

9 Schelstraete P, Deschaght P, Van Simaey L, et al. Genotype based evaluation of Pseudomonas aeruginosa eradication treatment success in cystic fibrosis patients. J Cyst Fibros 2010; 9: 99-103.

10 Hansen CR, Pressler T, Hoiby N. Early aggressive eradication therapy for intermittent Pseudomonas aeruginosa airway colonization in cystic fibrosis patients: 15 years experience. J Cyst Fibros 2008; 7: 523-530.

\section{Tuberculosis outbreak in East Greenland: groups at risk in an isolated arctic setting}

To the Editor:

In 2009, an unusually high number of tuberculosis (TB) cases were reported from a settlement (Settlement $\mathrm{X})$ in East Greenland. 4 years earlier, screening among schoolchildren had documented all children in this settlement to be free of Mycobacterium tuberculosis infection (MTI), whereas similar screenings had shown an MTI prevalence of $8 \%$ among schoolchildren in the rest of East Greenland [1]. The average TB incidence rate in East Greenland 5 years prior to the outbreak was 300 per 100000 populations (fig. 1) [2, 3].

The documentation of areas with differences in M. tuberculosis transmission prior to a TB outbreak provided a special opportunity to study the risk of TB in these settings that have an otherwise comparable population. The objective of the study was to estimate the risk of TB and MTI during an outbreak, and to evaluate whether individuals from a previously $M$. tuberculosis transmission-free environment exhibited particular risk.

We conducted a cohort study including all inhabitants living in East Greenland on January 1, 2008 (n=3541). Participants were followed until TB notification, death, emigration or December 31, 2012. A personal Civil Registration System (CRS) identifier given to Greenlandic citizens at birth combined data across public registries. The cohort was stratified by residency (Settlement X or the rest of East Greenland) at study entry.

Notification of TB to the National Board of Health is mandatory and the Greenlandic TB case definition follows that of the World Health Organization (WHO) $[4,5]$. A positive interferon- $\gamma$ release assay (IGRA) (QuantiFERON-TB Gold; Cellestis, Venlo, the Netherlands) defined an MTI case. IGRA results among the cohort participants were obtained from routine diagnostics, contact tracing or previously conducted population screening and projects $[1,6]$. Information on covariates was obtained from the CRS, TB notifications and medical records. A Greenlandic birthplace of both parents defined Greenlandic heritage.

Since 1955, all newborns have been offered bacille Calmette-Guérin (BCG) vaccination with national coverage rates of $90-99 \%$. However, the BCG vaccination programme was temporarily discontinued between 1991 and 1996, and children born in these years were considered unvaccinated [2, 6, 7].

Crude TB incidence rates (IR) were estimated as TB cases per 100000 person-years. Incidence rate ratios were estimated with Cox proportional hazard models with age as the underlying time axis and baseline hazard rate stratified by sex [8]. 